\title{
Reproducibility of retinal circulation measurements obtained using laser speckle flowgraphy-NAVI in patients with glaucoma
}

This article was published in the following Dove Press journal:

Clinical Ophthalmology

18 August 2011

Number of times this article has been viewed

\author{
Naoko Aizawa \\ Yu Yokoyama \\ Naoki Chiba \\ Kazuko Omodaka \\ Masayuki Yasuda \\ Takaaki Otomo \\ Masahiko Nakamura \\ Nobuo Fuse \\ Toru Nakazawa \\ Department of Ophthalmology, \\ Tohoku University Graduate School \\ of Medicine Sendai, Miyagi, Japan
}

Correspondence: Toru Nakazawa

Department of Ophthalmology, Tohoku University Graduate School of Medicine,

I-I Seiryo-machi, Aoba-ku, Sendai,

Miyagi 980-8574, Japan

Tel +8I 227177294

Fax +8I 227177298

Email ntoru@oph.med.tohoku.ac.jp
Background: Laser speckle flowgraphy (LSFG) enables noninvasive quantification of the retinal circulation in glaucoma patients. In this study, we tested the intrasession reproducibility of LSFG-NAVI, a modified LSFG technique.

Methods: Sixty-five eyes from 33 subjects (male $(M)$ :female $(F)=17: 16$ ) with a mean age of $49.4 \pm 11.2$ years were examined in this study. Two parameters indicating reproducibility - the coefficient of variation (COV) and the intraclass correlation coefficient (ICC) - were analyzed three times on the same day that mean blur rate (MBR) was measured using LSFG-NAVI. The sites analyzed were the retinal artery and vein, the optic disk, and the choroid. Following classification according to the Glaucoma Hemifield Test (GHT; SITA-Standard 30-2 program), the COV and ICC were examined in patients with $(\mathrm{GHT}+; 38$ eyes, $\mathrm{M}: \mathrm{F}=20: 18$, average age $48.9 \pm 12.8$ years) and without (GHT-; 27 eyes, M:F $=13: 14$, average age $50.1 \pm 8.7$ years) abnormal glaucomatous visual fields.

Results: For all subjects, the intrasession reproducibility of MBR in the optic disk (COV: 3.4 \pm 2.0 ; ICC: 0.95 ) and choroid (COV: $4.7 \pm 3.4$; ICC: 0.98 ) was excellent. The reproducibility for the retinal vein (COV: $8.4 \pm 5.6$, ICC: 0.90$)$ and retinal artery (COV: $10.9 \pm 9.9$, ICC: 0.9$)$ was moderate. MBRs in the optic disk had good reproducibility in both the GHT+ group (COV: $3.8 \pm 2.0$; ICC: 0.97 ) and the GHT- group (COV: $2.9 \pm 2.1$; ICC: 0.95). Local assessment of the optic disk in normal or glaucoma patients showed that the COVs of the quadrant optic disk areas were best in the temporal area of MBR (3.4\%, $4.2 \%$, respectively).

Conclusion: LSFG-NAVI showed favorable reproducibility in evaluation of retinal circulation of glaucoma patients, particularly in the optic disk and choroid.

Keywords: ocular circulation, reproducibility, optic nerve, retina

\section{Introduction}

Glaucoma affects more than 70 million people worldwide ${ }^{1,2}$ and is the second most frequent cause of blindness. ${ }^{1}$ The increase in lifespan worldwide has increased the number of individuals presenting with glaucoma and blindness. ${ }^{3}$ Although the pathogenesis of glaucoma remains unclear, one population-based study has suggested that the important risk factors for glaucoma are intraocular pressure (IOP), senescence, and myopia. ${ }^{4}$ Other factors such as vascular components may also contribute to optic nerve damage in glaucoma. An increasing body of evidence suggests that dysfunction of ocular microcirculation in the optic nerve influences the progression of glaucoma. ${ }^{5-7}$ The association between the degree of optic nerve damage and the defect area has also been demonstrated using fluorescein angiography (FAG) ${ }^{8-12}$ Decreased microcirculation in the optic nerve induced by intravitreal administration of endothelin-1 in the 
optic nerve has been shown to cause cupping in rabbits. ${ }^{13}$ These data suggest that disturbance of the microcirculation in the optic nerve head is related to the severity and progression of glaucoma.

As described above, microcirculation was examined using FAG, but it is difficult to quantify changes in microcirculation. Recently, devices that measure ocular circulation have become available. These devices assess the relationship between quantitative retinal or retrobulbar circulation and visual function using scanning laser Doppler flowmetry $(\mathrm{SLDF})^{14}$ and color Doppler imaging. ${ }^{15,16}$ Although they enable quantification of ocular circulation, it is difficult to capture the same place and reproduce the same image because of the small capture area. By contrast, laser speckle flowgraphy (LSFG) allows for noninvasive quantification of the microcirculation in the optic disk, choroid, and retinal vessels separately in living eyes. ${ }^{17}$ The machine is equipped with a fundus camera, a diode laser, an image sensor, an infrared charge-coupled device (CCD) camera, and a highresolution digital CCD camera. Squared blur rate (SBR), a parameter that yields a theoretically exact measurement of retinal microcirculation, is proportional to blood velocity and showed good correlation with blood flow parameters when measured with other instruments that assess the ocular blood flow. ${ }^{14,18}$ With LSFG, 5 seconds are needed to acquire images of the ocular circulation. The images are analyzed and the three sets of heartbeat data are combined to form a single color map depicting the distribution of the retinal circulation. The LSFG method is therefore suitable for monitoring the time course of changes in tissue circulation at the same site in the same eye at various intervals, ranging from seconds to months. ${ }^{14,17,19-22}$ In January 2008, LSFG-NAVI, a modified version of LSFG, was approved for use in Japan (Softcare Ltd, Fukuoka, Japan). In the LSFG-NAVI, the parameter was used with mean blur rate (MBR) with the newer CCD camera and the relationship between MBR and SBR was
"MBR $=2 \times \mathrm{SBR} " .{ }^{23}$ Recently some studies utilizing MBR for the evaluation of blood flow were reported. ${ }^{24,25}$

There have been no reports on the reproducibility of LSFG-NAVI. Reproducibility of any diagnostic instrument is important both for achieving diagnostic accuracy and for monitoring changes due to disease. The reproducibility of the Heidelberg Retina Tomograph (HRT) ${ }^{26}$ and the Stratus $\mathrm{OCT}^{27-30}$ has been confirmed in the literature, and these measures are considered clinically useful measures of glaucoma progression. Additional studies testing the reproducibility of MBR in normal subjects or glaucoma patients are needed before the LSFG-NAVI can be safely used as a tool for diagnosing and monitoring glaucoma. The purpose of this study was to test the reproducibility of ocular circulation measurements using LSFG-NAVI to determine intrasession reproducibility.

\section{Methods}

\section{Patient eligibility}

To evaluate intrasession reproducibility, we classified the patients into two groups using the Glaucoma Hemifield Test (GHT). Patients were categorized as within normal limits (control patients, GHT-) or outside normal limits (glaucoma patients, GHT+). The MBRs from the GHT+ patients (38 eyes; $\mathrm{M}: \mathrm{F}=20: 18$; average age $48.9 \pm 12.8$ years) and GHT- patients ( 27 eyes; M:F = 13:14; average age $50.2 \pm 8.7$ years) were used for analysis (Table 1 ). Twentyfour GHT+ patients were on topical antiglaucoma treatment. Glaucoma was diagnosed as glaucomatous optic neuropathy with corresponding glaucomatous visual field loss according to the Anderson-Pattela classification as follows: (1) a GHT outside normal limits; (2) a cluster of three or more non-edge points in a location typical of glaucoma, all of which are significantly lower on the pattern deviation plot at a $P<0.05$ level and one that is lower at a $P<0.01$ level; and (3) a corrected pattern standard deviation that is

Table I Demographic data for patients with or without glaucoma

\begin{tabular}{|c|c|c|c|}
\hline & $\mathrm{GHT}+(\mathrm{n}=38)$ & GHT- $(n=27)$ & $P$ value \\
\hline Number of subjects & 38 & 27 & \\
\hline Age (years) & $48.9 \pm 12.8$ & $50.2 \pm 8.7$ & 0.60 \\
\hline Gender (males:females) & $20 / 18$ & $14 / 13$ & 0.95 \\
\hline Visual acuity (logMAR) & $-0.02 \pm 0.15$ & $-0.06 \pm 0.06$ & 0.41 \\
\hline Spherical equivalent (D) & $-4.2 \pm 2.7$ & $-3.8 \pm 2.8$ & 0.59 \\
\hline $\mathrm{MD}(\mathrm{dB})$ & $-7.2 \pm 7.2$ & $-0.4 \pm 1.4$ & $<0.0001$ \\
\hline Intraocular pressure (mmHg) & $17.1 \pm 3.4$ & $17.5 \pm 5.2$ & 0.69 \\
\hline Systolic blood pressure $(\mathrm{mmHg})$ & $123.1 \pm 14.7$ & $125.1 \pm 13.8$ & 0.28 \\
\hline Diastolic blood pressure $(\mathrm{mmHg})$ & $74.5 \pm 14.2$ & $74.0 \pm 12.3$ & 0.88 \\
\hline Heart rate (per minutes) & $73.6 \pm 11.0$ & $70.2 \pm 9.9$ & 0.23 \\
\hline
\end{tabular}


significant at the $P<0.05$ level. Automated perimetry was performed with a Swedish interactive threshold algorithm (SITA)-standard strategy from the 30-2 program of the Humphrey Field Analyzer (Carl Zeiss Meditec, Dublin, CA). Reliability criteria included fixation losses and falsepositive and false-negative rates of less than $20 \%$. Patients were excluded if ocular diseases other than open-angle glaucoma were present, if systemic diseases affecting the visual fields were present, or if they had undergone intraocular surgery.

The procedures used conformed to the Principles of the Declaration of Helsinki, and approval for the analysis of the data was obtained from the Institutional Review Board of the Tohoku Graduate School of Medicine.

\section{Laser speckle flowgraphy}

Ophthalmic examinations including slit-lamp biomicroscopy, fundus evaluation, and Goldmann applanation tonometry were performed. After the examination, the pupils of each subject were dilated with $0.5 \%$ tropicamide and $0.5 \%$ phenylephrine hydrochloride. Blood pressure and IOP were measured after patients had rested for 10 minutes in a sitting position in a dark room, and then ocular circulation was assessed using LSFG-NAVI. To determine intrasession reproducibility, LSFG was performed three times within 10 minutes. The position of each subject's face was reset on the head holder for each examination. The centre of the captured image was set as the midpoint between the optic disk and the macula (Figure 1A and B). Previous eye position was recorded using LSFG Measure (v 6.64.00; Softcare Ltd, Kyushu, Japan), which enabled us to capture the same area in the following examination. In addition, LSFG-NAVI allowed the investigator to adjust the focus by looking at the live-capture image. If the focus of the image was not satisfactory, horizontal dark lines appeared on the live image. Three parameters of the MBR in the optic disk were calculated by the LSFG Analyzer software (v 3.0.47; Softcare Ltd, Kyushu, Japan). After we had identified the margin of the optic disk by hand using a round band, the software segmented out the vessel using the automated definitive threshold and analysis of all the mean of the MBRs (AM), the vessel mean (VM), and the tissue mean (TM) (Figure 1C). These parameters were also calculated for the quadrant area of the superior (S), inferior (I), temporal (T), and nasal areas (N) of the optic disk (Figure 1D). For the analysis of retinal arteries, retinal veins, and choroids, we used the rectangular band (Figure 1B). For accurate sampling of retinal arteries and retinal veins, it is better to set the region of interest (ROI) to more than 1000 pixels and place it on the
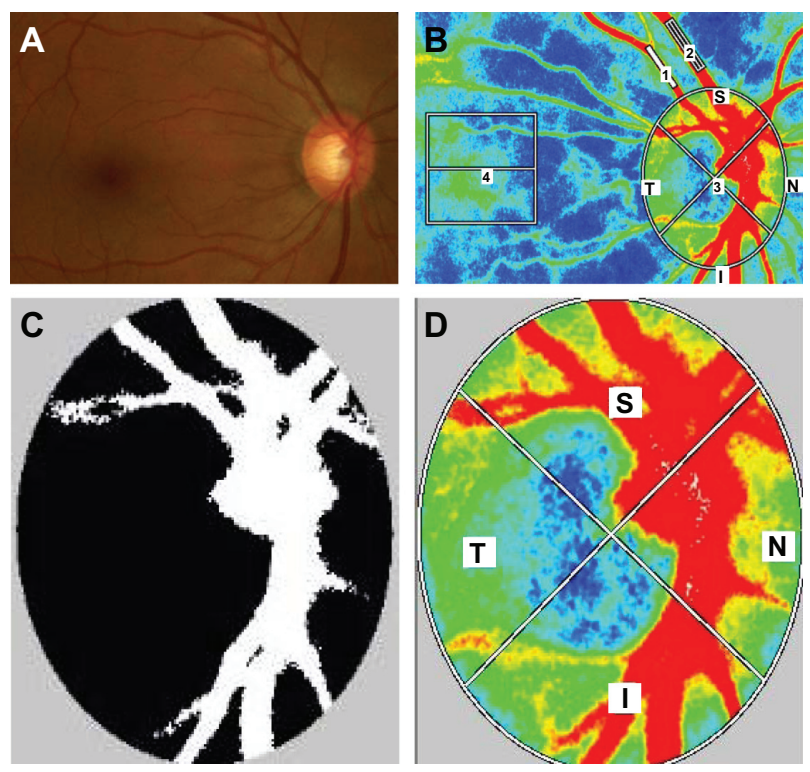

Figure I Representative photographs of a glaucoma patient in this study. (A) Photograph of the fundus. (B) A representative color map produced using LSFG (I, retinal artery; 2, retinal vein; 3, optic disk; 4, choroid). (C) Representative binary format images for segmentation between the vessel (white area) and tissue (black area), obtained using image viewer software with an automated definitive threshold. (D) Orientation of the quadrant assessment for the MBR in the optic disk.

vessel, avoiding inclusion of the nonvessel tissue. For analysis of the choroid, a square ROI $(150 \times 150$ pixels $)$ was set at one disk diameter away from the temporal margin of the disk without including the main retinal vessels (Figure 1B). All ROI positions were saved and used on the same patient for subsequent analyses.

\section{Analysis for reproducibility}

Two parameters indicating reproducibility - the coefficient of variation (COV) and the intraclass correlation coefficient (ICC) - were analyzed with the MBRs from LSFG-NAVI of three continuous examinations on the same day. The segments for analysis were set using a color map of the MBRs in the retinal artery (A), retinal vein (V), optic disk, and choroid.

\section{Statistical analyses}

Data were analyzed using statistical software Ekuseru-Toukei 2010 (Social Survey Research Information Co, Ltd, Tokyo, Japan). The Mann-Whitney U-test was used to compare two independent groups, and the chi-squared test was used for categorical data. A $P$-value of $<0.05$ was considered statistically significant.

\section{Results}

The first analysis was based on three examinations of 65 eyes (38 GHT+ eyes and 27 GHT- eyes) performed on the 
same day. The data from these examinations are summarized in Table 1. No significant differences in age, gender, visual acuity, or spherical equivalent (SE) were observed; however, there was a significant difference in mean deviation of Humphrey Field Analyzer (GHT+: -7.2 \pm 7.2 dB; GHT-: $-0.4 \pm 1.4 \mathrm{~dB} ; P<0.0001)$.

The intrasession reproducibility results of the measurements in the GHT-groups are summarized in Table 2. All COVs from these patients were less than $10 \%$, and all ICC results were more than 0.9 . The highest reproducibility was observed in the disk-VM (COV: 1.9\%; ICC: 0.98), and the lowest was observed in the MBRs of the retinal arteries (COV: 8.7\%; ICC: 0.9). The COVs for the disk parameters were excellent in both disk-AM and all the quadrant areas of the disk (inferior, $4.2 \%$; temporal, $3.4 \%$; superior, $3.6 \%$; nasal, 4.7\%).

Intrasession reproducibility of the measurements in the GHT+ groups is shown in Table 2. The highest reproducibility was observed in the disk-TM of the optic disk (COV: 2.3\%; ICC: 0.98), and the lowest reproducibility was observed in the retinal artery (COV: $12.4 \%$; ICC: 0.82).

In normal subjects, local assessment showed that the COVs of the quadrant optic disk areas were $3.6 \%$ in the S-AM, 3.4\% in the T-AM, $4.2 \%$ in the I-AM, and $4.7 \%$ in the N-AM. By contrast, the COVs of optic disk lesions were

Table 2 Intrasession reproducibility for GHT- and GHT+ subjects

\begin{tabular}{|c|c|c|c|c|}
\hline \multirow[t]{2}{*}{ MBR } & \multicolumn{2}{|l|}{ GHT- } & \multicolumn{2}{|l|}{ GHT+ } \\
\hline & cov & ICC & cov & ICC \\
\hline Artery & $8.7 \pm 4.5$ & 0.90 & $12.4 \pm 12.4$ & 0.82 \\
\hline Vein & $6.2 \pm 3.5$ & 0.90 & $10.1 \pm 6.3$ & 0.81 \\
\hline Choroid & $4.1 \pm 3.2$ & 0.98 & $5.2 \pm 3.7$ & 0.95 \\
\hline Disc-AM & $2.9 \pm 2.1$ & 0.95 & $3.8 \pm 2.0$ & 0.97 \\
\hline Disc-VM & $1.9 \pm 1.1$ & 0.98 & $2.6 \pm 1.8$ & 0.96 \\
\hline Disc-TM & $2.1 \pm 1.1$ & 0.97 & $2.3 \pm 1.5$ & 0.98 \\
\hline I-AM & $4.2 \pm 2.9$ & 0.98 & $5.8 \pm 4.1$ & 0.93 \\
\hline I-VM & $2.8 \pm 2.0$ & 0.98 & $3.5 \pm 1.9$ & 0.94 \\
\hline I-TM & $2.9 \pm 2.1$ & 0.97 & $3.5 \pm 2.7$ & 0.96 \\
\hline T-AM & $3.4 \pm 1.9$ & 0.99 & $4.2 \pm 2.3$ & 0.98 \\
\hline T-VM & $2.3 \pm 1.2$ & 0.93 & $4.2 \pm 3.8$ & 0.96 \\
\hline T-TM & $3.0 \pm 2.1$ & 0.98 & $3.7 \pm 2.3$ & 0.97 \\
\hline S-AM & $3.6 \pm 2.1$ & 0.98 & $6.4 \pm 4.4$ & 0.94 \\
\hline S-VM & $2.3 \pm 1.3$ & 0.98 & $3.5 \pm 2.8$ & 0.93 \\
\hline S-TM & $2.9 \pm 2.1$ & 0.96 & $4.6 \pm 2.9$ & 0.95 \\
\hline N-AM & $4.7 \pm 4.4$ & 0.92 & $5.1 \pm 4.2$ & 0.94 \\
\hline $\mathrm{N}-\mathrm{VM}$ & $2.3 \pm 1.7$ & 0.98 & $2.6 \pm 1.9$ & 0.97 \\
\hline N-TM & $3.6 \pm 2.5$ & 0.92 & $3.8 \pm 3.2$ & 0.95 \\
\hline
\end{tabular}

Abbrevations: AM, mean of all MBRs; COV, coefficient of variation; GHT, Glaucoma Hemifield Test; I, inferior quadrant area of optic disk; ICC, intraclass correlation coefficient; MBR, mean blur rate; N, nasal quadrant area of optic disk; S, superior quadrant area of optic disk; T, temporal quadrant area of optic disk; TM, tissue mean; VM, vessel mean.
$6.4 \%$ in the S-AM, $4.2 \%$ in the T-AM, $5.8 \%$ in the I-AM, and $5.1 \%$ in the N-AM in the glaucoma patients.

\section{Discussion}

Reproducibility of any objective devices for assessing glaucoma is important for both diagnosis and monitoring disease progression. A high level of reproducibility is also required for routine use of objective instruments because changes in the retinal microcirculation during the progression of glaucoma are normally small. Here, we used a modified LSFG to assess the microcirculation of the eyes of normal subjects and glaucoma patients.

The intrasession reproducibility of the three datasets captured on the same day was excellent, suggesting high reproducibility in both normal and glaucomatous eyes. In particular, the reproducibility of the measurements of the optic disk and choroid was excellent compared with the other areas of analysis (ie, the retinal artery and vein). The reproducibility of the retinal artery and vein measurements was significantly lower than that for the disk and choroid (Figure 1B). A wider area of analysis was associated with better reproducibility in MBR. In general, reliable reproducibility of the COV was less than $10 \%$, and the results of COVs of the optic disk (GHT-: 2.9\%; GHT+: $3.8 \%$ ) and choroid (GHT-: $4.1 \%$; GHT+: $5.2 \%$ ) were good. In the previous version of LSFG, the COVs of reproducibility were $11.7 \%$ in the optic disk and $8.7 \%$ in the choroid. These data suggest that the reproducibility of LSFG-NAVI is significantly improved compared to the previous $\mathrm{LSFG}^{31}$

Comparison of the COVs between GHT- and GHT+ showed that the COVs from the GHT- subjects were generally better than those of GHT+ subjects (Table 2). The COVs in the retinal vein, the I-VM, the S-AM, and the S-TM were high in the GHT+ subjects. In these areas, we should cautiously interpret the MBR data owing to the low degree of reproducibility among glaucoma patients. Hence, these data suggest that the intrasession reproducibility of LSFG was sufficient for evaluating ocular circulation in glaucoma patients in the clinic.

LSFG-NAVI enables evaluation of the MBR of the optic disk locally on the $\mathrm{S}, \mathrm{T}, \mathrm{I}$, and $\mathrm{N}$ quadrants and separately in the vascular and tissue area. Interestingly, we found that the reproducibility of the VM and TM in the optic disk was better than that of the overall optic disk mean (ie, the AM). In normal subjects, local assessment showed that the COVs of the quadrant optic disk areas were best in the T-AM and worst in the N-AM. By contrast, the COVs were best in the T-AM and worst in the S-AM in the glaucoma patients (Table 2). 
These data suggest that the reproducibility of the MBR decreased with structural changes due to glaucoma. The values of COVs in the glaucoma patients, however, were still sufficient for the evaluation of MBR in these patients. Upon further analysis of the VM and TM in local areas of the optic disk, the COV was generally better than that in the AM. These data suggest that the separation of the VM and TM in the optic disk was a good strategy for the assessment of disk microcirculation in the context of ocular disease.

There have been several reports on the reproducibility of objective glaucoma instruments. Budenz et $\mathrm{al}^{28,29}$ tested the reproducibility of retinal nerve fiber layer (RNFL) thickness measurements with Stratus-OCT (Carl Zeiss Meditec). They tested the intrasession variability of measurements between 88 normal subjects and 59 glaucoma patients and found that the ICCs ranged from 0.84 to 0.97 in normal subjects, with a range of COVs from $1.7 \%$ (mean RNFL) to $8.2 \%$ (nasal quadrant). In glaucomatous eyes, the ICCs ranged from 0.79 to 0.98 , with a COV range of $3.7 \%$ (mean RNFL) to $11.9 \%$ (nasal quadrant). Our results using the LSFG-NAVI suggest that the reproducibility of this instrument was better than that of the Stratus-OCT and that the LSFG-NAVI is a suitable instrument for measuring ocular circulation, with highly reproducible results.

OCT technology has improved significantly over the years, and various OCT devices are commercially available. These objective instruments require a high degree of reproducibility for purposes of glaucoma diagnosis. Menke et $\mathrm{al}^{32}$ tested the reproducibility of RNFL measurements of the 3D Fourier-Domain OCT in healthy volunteers and demonstrated an improvement in the reproducibility of RNFL measurements. Notably, the LSFG-NAVI analysis software already has the capacity to measure the same position. In general, in this study, the reproducibility of the MBR measurement of the vessels (retinal arteries and veins) was poorer than that for the disk and choroid. This deficit may be due to the small ROI. Furthermore, when the captured image had a small dark spot of vitreous opacity, it was difficult to determine the quality of the results after obtaining the color map of the MBR. To further improve the reproducibility of LSFG, attention should be focused on the chasing eye tracking technology used to follow eye movement, a high-resolution and high-contrast color map, and an index demonstrating the quality of the color map results.

In conclusion, the reproducibility of LSFG-NAVI was sufficient to evaluate the ocular circulation in this modified version of the LSFG owing to the improvement of the focus during capture. Reproducibility is important for the longitudinal assessment of the state of glaucoma patients.
We confirmed that LSFG-NAVI had significantly high reproducibility in measuring the microcirculation of the optic disk and choroid in both normal subjects and glaucoma patients. These data suggest that LSFG-NAVI is an effective and objective instrument for monitoring microcirculation. Although the role of microcirculation in glaucoma patients remains unclear, this technology yields highly reproducible results and could be used to explore the role of ocular circulation not only in glaucoma but also in other ocular diseases.

\section{Disclosure}

The authors report no conflicts of interest in this work.

\section{References}

1. Quigley HA. Number of people with glaucoma worldwide. $\mathrm{Br} J$ Ophthalmol. 1996;80(5):389-393.

2. Resnikoff $\mathrm{S}$, Pascolini $\mathrm{D}$, Etyàale $\mathrm{D}$, et al. Global data on visual impairment in the year 2002. Bull World Health Organ. 2004;82(11): 844-851.

3. Le A, Mukesh BN, McCarty CA, Taylor HR. Risk factors associated with the incidence of open-angle glaucoma: the visual impairment project. Invest Ophthalmol Vis Sci. 2003;44(9):3783-3789.

4. Suzuki Y, Iwase A, Araie M, et al. Risk factors for open-angle glaucoma in a Japanese population: the Tajimi Study. Ophthalmology. 2006; 113(9):1613-1617.

5. Caprioli J, Coleman AL. Blood pressure, perfusion pressure, and glaucoma. Am J Ophthalmol. 2010;149(5):704-712.

6. Venkataraman ST, Flanagan JG, Hudson C. Vascular reactivity of optic nerve head and retinal blood vessels in glaucoma - a review. Microcirculation. 2010;17(7):568-581.

7. Werne A, Harris A, Moore D, BenZion I, Siesky B. The circadian variations in systemic blood pressure, ocular perfusion pressure, and ocular blood flow: risk factors for glaucoma? Surv Ophthalmol. 2008;53(6): 559-567.

8. Arend O, Remky A, Plange N, Kaup M, Schwartz B. Fluorescein leakage of the optic disc in glaucomatous optic neuropathy. Graefes Arch Clin Exp Ophthalmol. 2005;243(7):659-664.

9. Plange N, Kaup M, Huber K, Remky A, Arend O. Fluorescein filling defects of the optic nerve head in normal tension glaucoma, primary open-angle glaucoma, ocular hypertension and healthy controls. Ophthalmic Physiol Opt. 2006;26(1):26-32.

10. Plange N, Kaup M, Weber A, Remky A, Arend O. Fluorescein filling defects and quantitative morphologic analysis of the optic nerve head in glaucoma. Arch Ophthalmol. 2004;122(2):195-201.

11. Sihota R, Saxena R, Taneja N, Venkatesh P, Sinha A. Topography and fluorescein angiography of the optic nerve head in primary openangle and chronic primary angle closure glaucoma. Optom Vis Sci. 2006;83(7):520-526.

12. Talusan ED, Schwartz B, Wilcox LM Jr. Fluorescein angiography of the optic disc. A longitudinal follow-up study. Arch Ophthalmol. 1980;98(9):1579-1587.

13. Sugiyama T, Mashima Y, Yoshioka Y, Oku H, Ikeda T. Effect of unoprostone on topographic and blood flow changes in the ischemic optic nerve head of rabbits. Arch Ophthalmol. 2009;127(4):454-459.

14. Yaoeda K, Shirakashi M, Funaki S, Funaki H, Nakatsue T, Abe H. Measurement of microcirculation in the optic nerve head by laser speckle flowgraphy and scanning laser Doppler flowmetry. $\mathrm{Am} \mathrm{J}$ Ophthalmol. 2000;129(6):734-739.

15. Yamazaki Y, Hayamizu F. Comparison of flow velocity of ophthalmic artery between primary open angle glaucoma and normal tension glaucoma. Br J Ophthalmol. 1995;79(8):732-734. 
16. YamazakiY, Drance SM. The relationship between progression of visual field defects and retrobulbar circulation in patients with glaucoma. Am J Ophthalmol. 1997;124(3):287-295.

17. Sugiyama T, Araie M, Riva CE, Schmetterer L, Orgul S. Use of laser speckle flowgraphy in ocular blood flow research. Acta Ophthalmol. 2010;88(7):723-729.

18. Nagahara M, Tamaki Y, Tomidokoro A, Araie M. In vivo measurement of blood velocity in human major retinal vessels using the laser speckle method. Invest Ophthalmol Vis Sci. 2011;52(1):87-92.

19. Tamaki Y, Kawamoto E, Araie M, Eguchi S, Fujii H. An application of laser speckle phenomenon for noninvasive two-dimensional evaluation of microcirculation in ocular fundus - a preliminary report. Jpn J Ophthalmol. 1993;37(2):178-186.

20. Tamaki Y, Araie M, Kawamoto E, Eguchi S, Fujii H. Noncontact, twodimensional measurement of retinal microcirculation using laser speckle phenomenon. Invest Ophthalmol Vis Sci. 1994;35(11):3825-3834.

21. Tamaki Y, Araie M, Kawamoto E, Eguchi S, Fujii H. Non-contact, two-dimensional measurement of tissue circulation in choroid and optic nerve head using laser speckle phenomenon. Exp Eye Res. 1995; 60(4):373-383.

22. Yaoeda K, Shirakashi M, Funaki S, et al. Measurement of microcirculation in optic nerve head by laser speckle flowgraphy in normal volunteers. Am J Ophthalmol. 2000;130(5):606-610.

23. Konishi N, Tokimoto Y, Kohra K, Fujii H. New laser speckle flowgraphy system using CCD camera. Opt Rev. 2002;9(4):163-169.

24. Watanabe G, Fujii H, Kishi S. Imaging of choroidal hemodynamics in eyes with polypoidal choroidal vasculopathy using laser speckle phenomenon. Jpn J Ophthalmol. 2008;52(3):175-181.
25. Liang Y, Downs JC, Fortune B, Cull G, Cioffi GA, Wang L. Impact of systemic blood pressure on the relationship between intraocular pressure and blood flow in the optic nerve head of nonhuman primates. Invest Ophthalmol Vis Sci. 2009;50(5):2154-2160.

26. Miglior S, Albe E, Guareschi M, Rossetti L, Orzalesi N. Intra- and interobserver reproducibility in the evaluation of the optic disc by HRT. Acta Ophthalmol Scand Suppl. 2002;236:45.

27. Anton A, Castany M, Pazos-Lopez M, Cuadrado R, Flores A, Castilla M. Reproducibility of measurements and variability of the classification algorithm of Stratus OCT in normal, hypertensive, and glaucomatous patients. Clin Ophthalmol. 2009;3:139-145.

28. Budenz DL, Chang RT, Huang X, Knighton RW, Tielsch JM. Reproducibility of retinal nerve fiber thickness measurements using the stratus OCT in normal and glaucomatous eyes. Invest Ophthalmol Vis Sci. 2005;46(7):2440-2443.

29. Budenz DL, Fredette MJ, Feuer WJ, Anderson DR. Reproducibility of peripapillary retinal nerve fiber thickness measurements with stratus OCT in glaucomatous eyes. Ophthalmology. 2008;115(4):661-666. e4.

30. Tzamalis A, Kynigopoulos M, Schlote T, Haefliger I. Improved reproducibility of retinal nerve fiber layer thickness measurements with the repeat-scan protocol using the Stratus OCT in normal and glaucomatous eyes. Graefes Arch Clin Exp Ophthalmol. 2009;247(2):245-252.

31. Tamaki Y, Araie M, Tomita K, Nagahara M, Tomidokoro A, Fujii H. Realtime measurement of human optic nerve head and choroid circulation, using the laser speckle phenomenon. Jpn J Ophthalmol. 1997;41(1):49-54.

32. Menke MN, Knecht P, Sturm V, Dabov S, Funk J. Reproducibility of nerve fiber layer thickness measurements using 3D fourier-domain OCT. Invest Ophthalmol Vis Sci. 2008;49(12):5386-5391.
Clinical Ophthalmology

\section{Publish your work in this journal}

Clinical Ophthalmology is an international, peer-reviewed journal covering all subspecialties within ophthalmology. Key topics include: Optometry; Visual science; Pharmacology and drug therapy in eye diseases; Basic Sciences; Primary and Secondary eye care; Patient Safety and Quality of Care Improvements. This journal is indexed on

\section{Dovepress}

PubMed Central and CAS, and is the official journal of The Society of Clinical Ophthalmology (SCO). The manuscript management system is completely online and includes a very quick and fair peer-review system, which is all easy to use. Visit http://www.dovepress.com/ testimonials.php to read real quotes from published authors. 